\title{
BMJ Open Sleep apnoea is a risk factor for Respiratory severe COVID-19
} Research

Satu Strausz, ${ }^{1,2,3}$ Tuomo Kiiskinen, ${ }^{1,4}$ Martin Broberg, ${ }^{1}$ Sanni Ruotsalainen, ${ }^{1}$ Jukka Koskela, ${ }^{1,5}$ Adel Bachour, ${ }^{6}$ FinnGen, Aarno Palotie, ${ }^{1,5,7}$ Tuula Palotie, ${ }^{2,3}$ Samuli Ripatti, ${ }^{1,5,8}$ Hanna M. Ollila ${ }^{1,5,9}$

To cite: Strausz S, Kiiskinen T, Broberg M, et al. Sleep apnoea is a risk factor for severe COVID-19. BMJ Open Resp Res 2021;8:e000845. doi:10.1136/ bmjresp-2020-000845

- Additional material is published online only. To view, please visit the journal online (http://dx.doi.org/10. 1136/bmjresp-2020-000845)

Received 25 November 2020 Revised 16 December 2020 Accepted 18 December 2020

\section{ABSTRACT}

Background Obstructive sleep apnoea (OSA) is associated with higher body mass index (BMI), diabetes, older age and male gender, which are all risk factors for severe COVID-19.

We aimed to study if OSA is an independent risk factor for COVID-19 infection or for severe COVID-19.

Methods OSA diagnosis and COVID-19 infection were extracted from the hospital discharge, causes of death and infectious diseases registries in individuals who participated in the FinnGen study ( $n=260405)$. Severe COVID-19 was defined as COVID-19 requiring hospitalisation. Multivariate logistic regression model was used to examine association. Comorbidities for either COVID-19 or OSA were selected as covariates. We performed a meta-analysis with previous studies. Results We identified 445 individuals with COVID-19, and $38(8.5 \%)$ of them with OSA of whom 19 out of $91(20.9 \%)$ were hospitalised. OSA associated with COVID-19 hospitalisation independent from age, sex, $\mathrm{BMI}$ and comorbidities ( $\mathrm{p}$-unadjusted $=5.13 \times 10^{-5}$, ORadjusted=2.93 (95\% Cl 1.02 to 8.39), p-adjusted $=0.045)$. OSA was not associated with the risk of contracting COVID-19 $(p=0.25)$. A meta-analysis of OSA and severe COVID-19 showed association across 15835 COVID-19 positive controls, and $n=1294$ patients with OSA with severe COVID-19 (OR=2.37 (95\% 1.14 to 4.95), $p=0.021$ ). Conclusion Risk for contracting COVID-19 was the same for patients with OSA and those without OSA. In contrast, among COVID-19 positive patients, OSA was associated with higher risk for hospitalisation. Our findings are in line with earlier works and suggest OSA as an independent risk factor for severe COVID-19.

\section{INTRODUCTION}

COVID-19 is a severe respiratory disease caused by SARS-CoV-2 virus infection. A subset of patients face hospitalisation, respiratory failure or even death. The severity of COVID-19 is highly age dependent but also evidenced by the number of individuals that receive hospital and intensive care treatment. ${ }^{12}$ Finland has had a relatively small number of COVID-19 cases in the spring of 2020. However, approximately 1\%-6\% of those tested positive for the virus in Finland received hospital or intensive care

\section{Key messages}

Is obstructive sleep apnoea (OSA) an independent risk factor for severe COVID-19?

- Patients with OSA have a higher risk to be hospitalised when affected by COVID-19 than non-OSA individuals.

> In assessment of patients with suspected or confirmed COVID-19 infection, OSA should be recognised as one of the comorbidity risk factors for developing a severe form of the disease and patients with OSA with suspected or confirmed COVID-19 infection should be monitored closely.

treatment. ${ }^{3}$ These percentages are similar to those reported globally. ${ }^{12}$ Severe COVID-19 outcome is mediated primarily through respiratory distress. ${ }^{45}$ Risk factors for severe COVID-19 have been identified as older age, male sex, obesity, diabetes, cardiovascular disease and poor lung function. ${ }^{6}$ In addition, other respiratory diseases have been listed as potential contributors for COVID-19 severity. Indeed, there are studies suggesting that obstructive sleep apnoea (OSA) may be a risk factor for severe COVID-19. ${ }^{7-12}$ Such risk would have substantial effect as OSA is a common disease affecting at least $8 \%$ of the population with higher prevalence in older age groups reaching to over $20 \%$ in individuals over 60 years of age. ${ }^{13}$ The disease aetiology of OSA is characterised by repetitive apnoea-hypopnea cycles during sleep causing shortness of breath which can be associated with sometime severe oxygen desaturation, sleep disruption and increase in systolic and diastolic blood pressure. ${ }^{14}$ The known risk factors for OSA include obesity, high age, male sex and craniofacial and upper airway structure variations and anomalies. ${ }^{15}$ Similarly, OSA is associated with increased risk for cardiovascular mortality, especially if not treated. Finally, it is essential to note that treatment exists for the majority of patients with OSA so that night-time breathing can be 
supported by continuous positive airway pressure (CPAP) or mandibular advancement device (MAD). This treatment substantially decreases the risk for cardiovascular events and death. ${ }^{16}{ }^{17}$ Recently, questions have been raised about whether OSA constitutes a high risk for COVID-19 infection or COVID-19 hospitalisation.

We specifically aimed at evaluating if OSA associates with the risk for severe COVID-19 infection independently of other potential risk factors including age, sex, body mass index (BMI), hypertension, diabetes (including type 1 and type 2 diabetes), coronary heart disease (CHD) asthma and chronic obstructive pulmonary disease (COPD), and also whether the risk for contracting COVID-19 is elevated among patients with OSA.

\section{METHODS}

Study sample set

FinnGen (https://www.finngen.fi/en) is a large biobank study that aims to genotype 500000 Finns including two types of biobank collections: (1) population-based epidemiological cohorts and (2) mostly disease-based collections in all University Hospitals in Finland (online supplemental table 1). FinnGen combines these data with longitudinal registry data that record healthcare events over the entire lifespan including the National Hospital Discharge Registry (available from 1968), the Causes of Death Registry (available from 1969), the National Infectious Diseases Registry (available from 1995) and the Medication Reimbursement Registry (available from 1995), all these using unique national personal identification codes, for the whole population of Finland for lifetime information since the start of each registry. FinnGen has harmonised data from these registries of 260405 Finnish individuals. Registry data were available from the beginning of the registry until 31 December 2018 and Infectious Registry data until 30 October 2020. In addition, demographic and anthropometric data of BMI and smoking status are included.

The information of COVID-19 positive individuals was collected from The National Infectious Diseases Registry and the infection was verified by laboratory test using PCR testing. Data for risk factors, comorbidities and OSA diagnosis were obtained from the National Hospital Discharge Registry, the Causes of Death Registry and the Medication Reimbursement Registry, where the clinical endpoints had been generated using International Classification of Diseases codes for OSA, hypertension, diabetes (including type 1 and type 2 diabetes), CHD, asthma and COPD (online supplemental table 2).

Treatment information concerning OSA was collected from the patient records of Heart and Lung Center or Department of Oral and Maxillofacial Diseases, Helsinki University Hospital (HUH), Finland.

The diagnosis in this special healthcare derived data for OSA is typically based on the following criteria: subjective symptoms, clinical examination and sleep registration applying Apnoea-Hypopnea Index (AHI) $5 \geq$ per hour for polysomnography or respiratory event index $5 \geq$ per hour for out-of-centre sleep study.

For the meta-analysis, estimates were collected from the previous studies ${ }^{7-9}$ concerning the role of OSA on COVID-19 hospitalisation.

\section{Statistical methods}

Differences in baseline demographics and clinical characteristics were tested using on $\chi^{2}$ tests. Fisher's exact test was used if the expected cell size was $\leq 5$. For continuous variables, Student's t-test was used. We considered $\mathrm{p}<0.05$ as statistically significant, and all tests were two sided (tables 1 and 2). All p values based on forementioned tests are Bonferroni corrected. Logistic regression was used to calculate OR between hospitalised and nonhospitalised groups. Model 1 was adjusted for age and

Table 1 Comparison of the baseline characteristics among COVID-19 positive individuals

\begin{tabular}{lllll}
\hline & All & Non-hospitalised $\mathbf{n = 3 5 4}$ & $\begin{array}{l}\text { Hospitalised } \\
\mathbf{n}=\mathbf{9 1}\end{array}$ & $\mathbf{P}_{\text {unadusted }}$ \\
\hline Age (mean in years, SD) & $52.7(17.4)$ & $49.3(16.3)$ & $65.9(14.8)$ & $1.06 \times 10^{-15^{*}}$ \\
Sex (male) (N, \%) & $166(37.3)$ & $133(37.6)$ & $33(36.3)$ & 1 \\
\hline OSA (N, \%) & $38(8.5)$ & $19(5.4)$ & $19(20.9)$ & $5.13 \times 10^{-5^{*}}$ \\
\hline BMI (mean kg/m², SD) & $27.13(5.44)$ & $26.54(5.20)$ & $29.25(5.78)$ & $0.014^{*}$ \\
\hline Hypertension (N, \%) & $79(17.8)$ & $40(11.3)$ & $39(42.9)$ & $5.03 \times 10^{-11^{*}}$ \\
\hline Diabetes (N, \%) & $46(10.3)$ & $23(6.5)$ & $23(25.3)$ & $3.45 \times 10^{-6^{*}}$ \\
CHD (N, \%) & $21(4.7)$ & $9(2.5)$ & $12(13.2)$ & $5.20 \times 10^{-4^{*}}$ \\
\hline Asthma/COPD & $54(12.1)$ & $40(11.3)$ & $14(15.4)$ & 1 \\
\hline
\end{tabular}

Differences and associations between non-hospitalised and hospitalised COVID-19 positive individuals. Baseline demographics and clinical characteristics $\mathrm{P}_{\text {unadjusted }}$ values were based on $\chi^{2}$ test. For continuous variables, we used Student's t-test. BMI was measured of 264 participants including 206 non-hospitalised and 58 hospitalised individuals.

*Statistically significant.

BMI, body mass index; CHD, coronary heart disease; COPD, chronic obstructive pulmonary disease; OSA, obstructive sleep apnoea. 
Table 2 Description of patients with COVID-19 with or without obstructive sleep apnoea (OSA) and a comparison between non-hospitalised and hospitalised patients with OSA

\begin{tabular}{|c|c|c|c|c|c|c|}
\hline & $\begin{array}{l}\text { Non-OSA } \\
n=407\end{array}$ & $\begin{array}{l}\text { OSA } \\
\mathrm{n}=38\end{array}$ & $P$ value & $\begin{array}{l}\text { OSA } \\
\text { Non-hospitalised } \\
n=19\end{array}$ & $\begin{array}{l}\text { OSA } \\
\text { hospitalised } \\
n=19\end{array}$ & $P$ value \\
\hline Age (mean in years, SD) & $51.9(17.5)$ & $61.3(12.9)$ & $5.60 \times 10^{-4^{*}}$ & $56.3(11.0)$ & $66.3(12.9)$ & 0.057 \\
\hline Sex (male) (N, \%) & 147 (36.1) & $19(50.0)$ & 0.645 & 12 (63.2) & $7(36.8)$ & 0.776 \\
\hline BMI (mean $\mathrm{kg} / \mathrm{m}^{2}, \mathrm{SD}$ ) & $26.71(5.27)$ & $31.15(5.56)$ & $3.38 \times 10^{-3^{*}}$ & $30.91(5.58)$ & $31.37(5.77)$ & 1 \\
\hline $\begin{array}{l}\text { Comorbidities or outcomes } \\
(\mathrm{N}, \%)\end{array}$ & $118(29.0)$ & 24 (63.2) & $1.74 \times 10^{-4^{*}}$ & $12(63.2)$ & 12 (63.2) & 1 \\
\hline Hospitalised (N, \%) & $72(17.7)$ & $19(50.0)$ & \multicolumn{4}{|l|}{$3.21 \times 10^{-5^{*}}$} \\
\hline $\begin{array}{l}\text { Differences between non-OSA } \\
\text { COVID-19-positive individuals. } \\
\text { values were based on } \chi^{2} \text { test. Fi } \\
\text { was measured of } 264 \text { participar } \\
\text { coronary heart disease, asthma } \\
\text { *Statistically significant. } \\
\text { BMI, body mass index. }\end{array}$ & $\begin{array}{l}\text { 7/19 patients } \\
\text { Fisher's exact } \\
\text { nts including } 2 \\
\text { a, chronic obst }\end{array}$ & $\begin{array}{l}\text { ospitalisation } \\
\text { ns used if the } \\
\text { n-OSA and } 25 \\
\text { pulmonary }\end{array}$ & $\begin{array}{l}\text { OSA diagn } \\
\text { ple size wa } \\
\text { A individua } \\
\text { se. }\end{array}$ & $\begin{array}{l}\text { th did not have any } \\
\text { morbidities and outc }\end{array}$ & $\begin{array}{l}\text { ed patients wit } \\
\text { disease comor } \\
\text { we used Stude } \\
\text { =hypertension }\end{array}$ & $\begin{array}{l}\text { SA among } \\
\text { ties P } \\
\text { t-test. BM } \\
\text { abetes, }\end{array}$ \\
\hline
\end{tabular}

sex. Model 2 was adjusted for BMI in addition to covariates of Model 1. Model 3 was adjusted for BMI, hypertension, diabetes, CHD, asthma and COPD in addition to covariates of Model 1 (table 3) and similarly between COVID-19 -positive and non-COVID-19 groups .

Our results and previous findings from the corresponding studies $^{7-9}$ were pooled together forming a meta-analysis using restricted maximum likelihood estimation of the random effect model in the Metagen $\mathrm{R}$. The merged data consisted of 15835 COVID-19 positive individuals including 1294 patients with OSA.

The R statistical package (V.4.0.2) was used for all analyses (www.r-project.org)

\section{Patient and public involvement}

Patients and public were not involved in the designing process of this study. The patients will not be informed individually of the study results otherwise than through possible media coverage.

\section{RESULTS}

The data included 260405 Finnish individuals from FinnGen Data Freeze 6 with 445 patients with COVID-19.

\begin{tabular}{|c|c|c|c|}
\hline & OR & $95 \% \mathrm{Cl}$ & $P$ value \\
\hline Model 1 & 3.85 & 1.82 to 8.13 & $4.13 \times 10^{-4^{*}}$ \\
\hline Model 2 & 3.45 & 1.27 to 9.35 & $0.016^{\star}$ \\
\hline Model 3 & 2.93 & 1.02 to 8.39 & $0.045^{*}$ \\
\hline
\end{tabular}

Model 1 is adjusted for age and sex. Model 2 is adjusted for body mass index (BMI) in addition to covariates of Model 1. Model 3 is adjusted for BMI, hypertension, diabetes, coronary heart disease, asthma and chronic obstructive pulmonary disease in addition to covariates of model 1.

*Statistically significant.
Of them, $38(8.5 \%)$ had OSA. Severe COVID-19 cases $(\mathrm{n}=91)$ included $19(20.9 \%)$ patients with OSA. Severe COVID-19 was defined as an infection requiring hospitalisation.

Of all patients with COVID-19 diagnosis $(\mathrm{n}=445,37.3 \%$ male, mean age 52.7 years) 38 patients also had OSA diagnosis $(8.5 \%, 50.0 \%$ male, mean age 61.3 years), (tables 1 and 2). This reflects a similar prevalence of OSA diagnoses in COVID-19 infected as in the normal population in FinnGen, where prevalence is $8 \% .{ }^{18}$ Ninety-one $(20.4 \%)$ patients required hospitalisation $(36.3 \%$ male, mean age 65.9 years) including 19 patients with OSA (table 1).

Prevalence of OSA $\left(\mathrm{p}=5.13 \times 10^{-5}\right)$, hypertension $\left(\mathrm{p}=5.03 \times 10^{-11}\right)$, diabetes $\left(\mathrm{p}=3.45 \times 10^{-6}\right)$ and CHD $\left(\mathrm{p}=5.20 \times 10^{-4}\right)$ were statistically significantly higher in the hospitalised group. Similarly, age and BMI were higher among hospitalised individuals $\left(\mathrm{p}=1.06 \times 10^{-15}, \mathrm{p}=0.014\right.$, respectively; table 1 ).

To evaluate the performance of the diagnostic events, we compared the main risk factors among patients with COVID-19 between individuals with OSA $(n=38)$ and those who did not have OSA diagnosis $(n=407)$. Patients with OSA were statistically significantly older and their BMI was higher (mean age 61.3 years, BMI $31.15 \mathrm{~kg} / \mathrm{m}^{2}$, $\mathrm{p}=5.60 \times 10^{-4}, \mathrm{p}=3.38 \times 10^{-3}$, respectively) than non-OSA individuals (mean age 51.9 years, BMI $26.71 \mathrm{~kg} / \mathrm{m}^{2}$ ). Also, comorbidities were more prevalent among OSA individuals $\left(\mathrm{p}=1.74 \times 10^{-4}\right)$ and they faced hospitalisation more often $\left(p=3.21 \times 10^{-5}\right)$. We did not observe differences in risk factors when comparing non-hospitalised $(\mathrm{n}=19$, male $63.2 \%$, mean age 56.3 years $)$ and hospitalised patients with OSA $(\mathrm{n}=19$, male $36.8 \%$, mean age 66.3 years; table 2). Furthermore, $7 / 19$ patients with OSA were hospitalised due to COVID-19 did not have any other disease comorbidities. We did not observe significant differences concerning age or BMI between individuals who had only OSA as a comorbidity or also 


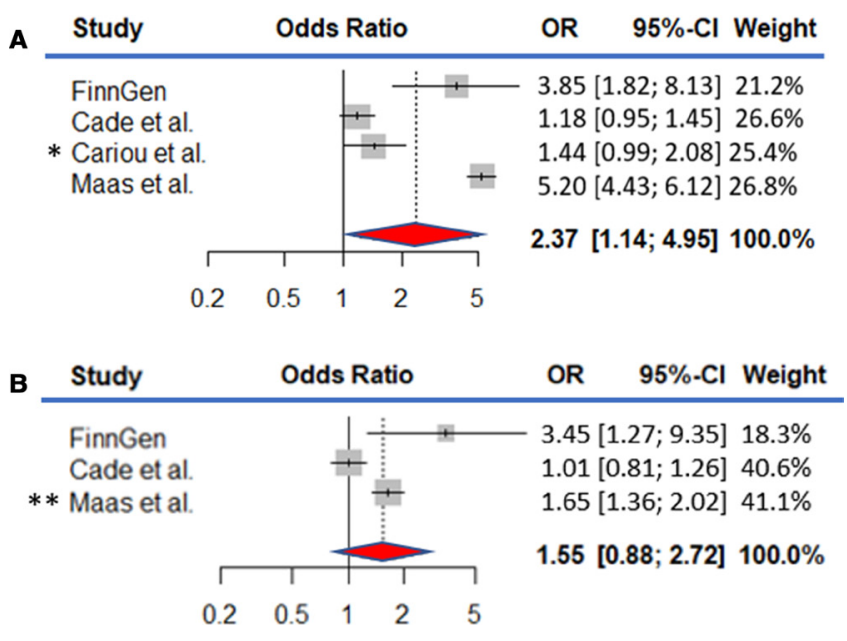

Figure 1 Forest plots of obstructive sleep apnoea (OSA) and the risk of hospitalisation due to COVID-19 The evidence is combined using random-effect meta-analysis. The data consisted of 15835 COVID-19 positive individuals including 1294 patients with OSA. (A) The model is adjusted for age and sex, and ethnic background if available. ${ }^{*}$ Cariou et al study's primary outcome was defined as tracheal intubation and/or death within 7 days of admission. (B) The model is adjusted for age, sex and BMI. ${ }^{* *}$ Maas et al study is adjusted for diabetes and hypertension in addition to forementioned covariates.

other comorbidities in addition to OSA (62.8 years, 68.9 years, $\mathrm{p}=0.72,30.26 \mathrm{~kg} / \mathrm{m}^{2}, 32.67 \mathrm{~kg} / \mathrm{m}^{2}, \mathrm{p}=1.00$, respectively). Time since the first diagnosis of OSA to COVID-19 infection was not significantly different between nonhospitalised and hospitalised groups (6.6 years, 8.6 years, $\mathrm{p}=0.37$, respectively).

We tested if OSA is associated with the risk of severe COVID-19 infection, determined as being hospitalised $(n=91)$, or COVID-19 infection in general $(n=445)$. While OSA did not affect the risk of contracting COVID-19 $(p=0.25)$, patients with OSA had a considerably elevated risk for being hospitalised due to severe COVID-19 (OR=2.93 (95\% CI 1.02 to 8.39), $\mathrm{p}=0.045$, adjusted for age, sex, BMI, hypertension, diabetes, CHD, asthma and COPD; table 3).

We performed a meta-analysis by pooling the comparable endpoints between the published studies ${ }^{7-9}$ and examined the association with severe COVID-19 with and without adjusting for BMI. The studies, including our study, consisted of 15835 COVID-19 positive individuals including 1294 patients with OSA. Overall, we observed over twofold increase in the pooled estimate prior to adjusting for BMI (OR=2.37 (1.14 to 4.95), $\mathrm{p}=0.021$ ). Adjusted estimates were available in the study of Cade et $a l^{8}$ (BMI) and Maas et a ${ }^{\ominus}$ (BMI, hypertension and diabetes) but not in the study of Cariou et al. ${ }^{7}$ The pooled estimate somewhat attenuated after adjustment for BMI ( $\mathrm{OR}=1.55$ (0.88 to 2.72), $\mathrm{p}=0.13$; figure 1 ).

To investigate patients with OSA contracted with COVID-19 in more detail, we accessed the healthcare data of 305 COVID-19 positive individuals including 11
Table 4 Healthcare data characteristics of individuals with obstructive sleep apnoea contracted with COVID-19

$\mathrm{n}=11$

\begin{tabular}{|c|c|}
\hline Age (mean in years) & $55.1(8.0)$ \\
\hline Sex (male) $(N, \%)$ & $9(81.8)$ \\
\hline $\mathrm{BMI}\left(\right.$ mean $\left.\mathrm{kg} / \mathrm{m}^{2}, \mathrm{SD}\right)$ & $35.08(5.96)$ \\
\hline Diabetes (N, \%) & $3(27.3)$ \\
\hline Hypertension (N, \%) & 7 (63.6) \\
\hline $\mathrm{CHD}(\mathrm{N}, \%)$ & $3(27.3)$ \\
\hline CPAP (N, \%) & $7(63.6)$ \\
\hline MAD (N, \%) & $1(9.1)$ \\
\hline AHI (mean, events per hour) & $43.3(20.6)$ \\
\hline ODI (mean, events per hour) & $39.6(15.7)$ \\
\hline $\mathrm{SpO}_{2}$ mean (mean $\left.\%, \mathrm{SD}\right)$ & $91.0(3.3)$ \\
\hline $\mathrm{SpO}_{2} \min ($ mean $\%, \mathrm{SD})$ & $79.4(8.3)$ \\
\hline AHI with CPAP (mean, events per hour) & $1.86(2.07)$ \\
\hline $\operatorname{ICU}(\mathrm{N}, \%)$ & $3(27.3)$ \\
\hline NIV (N, \%) & $11(100)$ \\
\hline Intubation (N, \%) & $2(18.2)$ \\
\hline Treatment time in hospital (mean in days, SD) & $15(8.6)$ \\
\hline
\end{tabular}

AHI, Apnoea-Hypopnea Index; BMI, body mass index; CHD, coronary heart disease; CPAP, continuous positive airway pressure; ICU, intensive care unit; MAD, mandibular advancement devise; NIV, non-invasive ventilation; ODI, oxygen desaturation index; SpO2, oxygen saturation.

patients with OSA treated in Heart and Lung Center or Department of Oral and Maxillofacial Diseases, HUH, Finland by the end of October 2020. The mean age of the COVID-19 positive patients with OSA was 55.1 years, mean BMI was $35.08 \mathrm{~kg} / \mathrm{m}^{2}$ and $9(81.8 \%)$ patients were male. Mean AHI was 43.3 events per hour (table 4 ) and all 11 patients had either moderate $(\mathrm{AHI} \geq 15$ but $<30$ events per hour) or severe (AHI $\geq 30$ events per hour) OSA; 9 out of 11 had severe OSA and 2 out of 11 had moderate OSA. 8/11 had treated OSA; 7 patients had CPAP therapy and 1 patient had MAD. CPAP-treated patients used their appliances $98 \%$ of the nights with 6.3 hours mean time used. Mean AHI during treatment was 1.86 events/hour prior to the COVID-19 infection. Despite of the OSA treatment, all 11 patients required hospital treatment caused by pneumonia or other COVID-19 caused symptoms, such as high fewer. All patients needed non-invasive ventilation and two required intubation. Treatment time in hospital was 15 days (mean, table 4).

\section{DISCUSSION}

Here, we examined the role of OSA as a risk factor for COVID-19 leading to hospitalisation. Our analyses revealed 2.93-times higher risk for COVID-19 hospitalisation in patients with OSA, independently of BMI and other known risk factors for OSA, or those for severe 
COVID-19 suggesting that OSA is an independent risk factor for COVID-19.

Indeed, our findings are well in line with earlier works on COVID-19 comorbidities and OSA. Three studies have examined the association between COVID-19 and OSA before. Despite different endpoint definitions (contracting COVID-19, severity of the disease, mechanical ventilation, and death), these studies share similar findings with ours. All studies showed a significant association with COVID-19 severity and OSA. ${ }^{7-9}$ However, only one study showed a statistically significant association between OSA and severe COVID-19 after adjusting for BMI. ${ }^{9}$ These findings suggest that while OSA is likely a risk factor for COVID-19, evaluating the magnitude of this association would benefit from harmonised analyses across different cohorts where comorbidities are similarly assessed.

Building on these studies, we set to test the role of OSA on COVID-19 hospitalisation. In our study, patients with OSA had 2.93 times higher risk of being hospitalised and the estimate was comparable and independent of the risk in diabetes patients, where elevated risk has been reported earlier for severe COVID-19. ${ }^{19}$ There are at least two potential pathological mechanisms how OSA may relate to severe COVID-19. First, individuals with OSA often have one or more comorbidities that are known risk factors for severe COVID-19. For example, high BMI increases the risk for severe COVID-19. Furthermore, OSA exacerbates the effects of many underlying risk factors increasing blood pressure. Second, OSA may worsen the core symptoms of severe COVID-19, especially during the night, when decreased oxygen saturation levels occur in OSA. ${ }^{14}$ Our findings together with earlier reports suggest that OSA should be taken into account when assessing who will develop life-threatening complications of COVID-19 infection.

In addition, we collected treatment information concerning OSA of 11 patients who had contracted COVID-19. Despite of the OSA treatment, patients developed a severe form of COVID-19 and all patients required hospital care suggesting OSA as a risk factor even if treated.

Finally, based on our results and previous studies, ${ }^{7-9}$ we set a meta-analysis to strengthen the role of OSA on COVID-19 hospitalisation. We were able to establish OSA as a risk factor and show that the effect is related to COVID-19 severity indicating over 2-fold risk.

Our findings should be interpreted in the context that registry-based ascertainment through hospitalisation may miss non-hospitalised OSA cases (false negatives). In addition, the population prevalence of OSA is larger than the observed prevalence as OSA is still underdiagnosed in Finland. This may affect to our findings. The high percentage of individuals in hospitals likely reflect the age distribution of the first wave patients in Finland, where infections took place in individuals approximately 50 years of age, and selective testing in those that had clear or severe symptoms. Similarly, early on the testing was targeted towards older individuals and to those with comorbidities, as not enough testing capacity was available early in the spring 2020. Although sufficient capacity and different strategies are in place now, the number of patients with mild COVID-19 infection may have not been recorded in the registries from the early spring 2020. Finally, compared with other countries there have been relatively small numbers of COVID-19 infections in Finland till the end of October 2020 when these data were curated. Therefore, while the effect estimates are comparable to other studies on OSA and COVID-19, the confidence intervals are relatively large due to the smaller total number of COVID-19 positive individuals in Finland and in the study sample: less than 500 patients compared with several hundred in other studies. Also, FinnGen represents older population (mean 58.6 years) than the average age (mean 42.9 years) in Finland. ${ }^{20}$

In conclusion, patients with OSA have the same risk of contracting COVID-19 than non-OSA individuals. Meanwhile, in this study, patients with OSA had 2.93 times higher risk to be hospitalised when affected by COVID-19 than non-OSA individuals. Our findings may suggest that, in assessment of patients with suspected or confirmed COVID-19 infection, OSA should be recognised as one of the comorbidity risk factors for developing a severe form of the disease. We believe that our finding may help in identifying high-risk individuals for severe forms of COVID-19 infection and therefore screening for previous indications of OSA could be beneficial among individuals testing positive for the virus.

\section{Author affiliations}

${ }^{1}$ Institute for Molecular Medicine Finland (FIMM), University of Helsinki, Helsinki, Finland

${ }^{2}$ Orthodontics, Department of Oral and Maxillofacial Diseases, Clinicum, Faculty of Medicine, University of Helsinki, Helsinki, Finland

${ }^{3}$ Department of Oral and Maxillofacial Diseases, Helsinki University Hospital (HUH), Helsinki, Finland

${ }^{4}$ Finnish Institute for Health and Welfare, Helsinki, Finland

${ }^{5}$ Broad Institute of MIT and Harvard, Cambridge, MA, USA

${ }^{6}$ Sleep Unit, Heart and Lung Center, Helsinki University Hospital (HUH), Helsinki, Finland

${ }^{7}$ Analytic and Translational Genetics Unit (ATGU), Department of Medicine, Department of Neurology and Department of Psychiatry, Massachusetts General Hospital, Boston, Massachusetts, USA

${ }^{8}$ Department of Public Health, University of Helsinki, Helsinki, Finland

${ }^{9}$ Stanford University School of Medicine, Palo Alto, CA, USA

Correction notice This article has been corrected since it first published. The provenance and peer review statement has been included.

Acknowledgements The authors would like to thank all participants of the FinnGen study for their generous participation. Patients and control subjects in FinnGen provided informed consent for biobank research, based on the Finnish Biobank Act. Alternatively, older research cohorts, collected prior the start of FinnGen (in August 2017), were collected based on study-specific consents and later transferred to the Finnish biobanks after approval by Fimea, the National Supervisory Authority for Welfare and Health. Recruitment protocols followed the biobank protocols approved by Fimea. The Coordinating Ethics Committee of the Hospital District of Helsinki and Uusimaa (HUS) approved the FinnGen study protocol Nr HUS/990/2017.

Contributors $\mathrm{HO}$ is the guarantor of the manuscript. TK, HO, SR and SS conceived the study and designed the study protocol. TK, HO and SS conducted the literature review, statistical analysis and drafted the manuscript. MB and SRu contributed statistical analysis and TK phenotyped study samples. AB, MB, JK, SR, SRu, AP, TP 
and SR reviewed the manuscript for intellectual content, made revisions as needed and approved the final version for publication. HO, TP and SR supervised the study.

Funding SR was supported by the Academy of Finland Center of Excellence in Complex Disease Genetics (Grant No 312062), the Finnish Foundation for Cardiovascular Research, the Sigrid Juselius Foundation and University of Helsinki HiLIFE Fellow and Grand Challenge grants and Juho Vainio Foundation \& Academy of Finland Covid-19 research funding. AP was supported by the Academy of Finland Center of Excellence in Complex Disease Genetics (Grant No 312074), and the Sigrid Juselius Foundation. HMO was supported by the Academy of Finland (Grant No 309643, 340539), Oskar Öfflund foundation, Yrjö Jahnsson foundation, Signe and Ane Gyllenberg foundation and Instrumentarium science foundation and TP by the HUCH research grant. The FinnGen project is funded by two grants from Business Finland (HUS 4685/31/2016 and UH 4386/31/2016) and the following industry partners: AbbVie, AstraZeneca UK, Biogen MA, Celgene Corporation, Celgene International II Sàrl, Genentech, Merck Sharp \& Dohme Corp, Pfizer, GlaxoSmithKline Intellectual Property Development, Sanofi US Services, Maze Therapeutics, Janssen Biotech. Following biobanks are acknowledged for the project samples: Auria Biobank (https://www.auria.fi/biopankki/en/), THL Biobank (https://thl.fi/en/web/thl-biobank), Helsinki Biobank (https://www. helsinginbiopankki.fi/en/home), Biobank Borealis of Northern Finland (https://www. ppshp.fi/Tutkimus-ja-opetus/Biopankki/Pages/Biobank-Borealis-briefly-in-English. aspx), Finnish Clinical Biobank Tampere (https://www.tays.fi/en-US/Research_ and development/Finnish Clinical Biobank Tampere), Biobank of Eastern Finland (https://ita-suomenbiopankki.fi/en/), Central Finland Biobank (https://www.ksshp.fi/ fi-Fl/Potilaalle/Biopankki), Finnish Red Cross Blood Service Biobank (https://www. bloodservice.fi/Research_Projects/biobanking) and Terveystalo Biobank (https:// www.terveystalo.com/fi/Yritystietoa/Terveystalo-Biopankki/Biopankki/). All Finnish Biobanks are members of BBMRI.fi infrastructure (http://www.bbmri.fi/).

\section{Competing interests None declared.}

Patient consent for publication Obtained.

Ethics approval The data collection from Heart and Lung Center or Department of Oral and Maxillofacial Diseases, Helsinki University Hospital (HUH) was approved under approval number HUS/141/2020. The FinnGen study is approved by Finnish Institute for Health and Welfare (permit numbers: THL/2031/6.02.00/2017, THL/1101/5.05.00/2017, THL/341/6.02.00/2018, THL/2222/6.02.00/2018, THL/283/6.02.00/2019, THL/1721/5.05.00/2019, THL/1524/5.05.00/2020, and THL/2364/14.02/2020), Digital and population data service agency (permit numbers: VRK43431/2017-3, VRK/6909/2018-3, VRK/4415/2019-3), the Social Insurance Institution (permit numbers: KELA 58/522/2017, KELA 131/522/2018, KELA 70/522/2019, KELA 98/522/2019, KELA 138/522/2019, KELA 2/522/2020, KELA 16/522/2020 and Statistics Finland (permit numbers: TK-53-1041-17 and TK-53-90-20). The Biobank Access Decisions for FinnGen samples and data used in FinnGen Data Freeze 6 include: THL Biobank BB2017_55, BB2017_111, BB2018_19, BB_2018_34, BB_2018_67, BB2018_71, BB2019_7, BB2019_8, BB2019_26, BB2020_1, Finnish Red Cross Blood Service Biobank 7.12.2017, Helsinki Biobank HUS/359/2017, Auria Biobank AB17-5154, Biobank Borealis of Northern Finland_2017_1013, Biobank of Eastern Finland 1186/2018, Finnish Clinical Biobank Tampere MH0004, Central Finland Biobank 1-2017, and Terveystalo Biobank STB 2018001.

Provenance and peer review Not commissioned; externally peer reviewed.

Data availability statement Data are available upon reasonable request. The FinnGen individual level data may be accessed through applications to the Finnish Biobanks' FinnBB portal, Fingenious (www.finbb.fi). Summary data can be accessed through the FinnGen site https://www.finngen.fi/en/access_results.

Supplemental material This content has been supplied by the author(s). It has not been vetted by BMJ Publishing Group Limited (BMJ) and may not have been peer-reviewed. Any opinions or recommendations discussed are solely those of the author(s) and are not endorsed by BMJ. BMJ disclaims all liability and responsibility arising from any reliance placed on the content. Where the content includes any translated material, BMJ does not warrant the accuracy and reliability of the translations (including but not limited to local regulations, clinical guidelines, terminology, drug names and drug dosages), and is not responsible for any error and/or omissions arising from translation and adaptation or otherwise.
Open access This is an open access article distributed in accordance with the Creative Commons Attribution Non Commercial (CC BY-NC 4.0) license, which permits others to distribute, remix, adapt, build upon this work non-commercially, and license their derivative works on different terms, provided the original work is properly cited, appropriate credit is given, any changes made indicated, and the use is non-commercial. See: http://creativecommons.org/licenses/by-nc/4.0/.

\section{REFERENCES}

1 Garg S, Kim L, Whitaker M, et al. Hospitalization Rates and Characteristics of Patients Hospitalized with Laboratory-Confirmed Coronavirus Disease 2019 - COVID-NET, 14 States, March 1-30, 2020. MMWR Morb Mortal Wkly Rep 2020;69:458-64.

2 Salje H, Tran Kiem C, Lefrancq N, et al. Estimating the burden of SARS-CoV-2 in France. Science 2020;369:208-11.

3 Finnish Institute for Health and Welfare. Infectious diseases and vaccinations. Available: https://thl.fi/en/web/infectious-diseasesand-vaccinations/what-s-new/coronavirus-covid-19-latest-updates/ situation-update-on-coronavirus

$4 \mathrm{Li} \mathrm{X,} \mathrm{Ma} \mathrm{X.} \mathrm{Acute} \mathrm{respiratory} \mathrm{failure} \mathrm{in} \mathrm{COVID-19:} \mathrm{is} \mathrm{it} \mathrm{"typical"}$ ARDS? Crit Care 2020;24:198-020.

5 Fan E, Beitler JR, Brochard L, et al. COVID-19-associated acute respiratory distress syndrome: is a different approach to management warranted? Lancet Respir Med 2020;8:816-21.

6 Jordan RE, Adab P, Cheng KK. Covid-19: risk factors for severe disease and death. BMJ 2020;368:m1198.

7 Cariou B, Hadjadj S, Wargny M, et al. Phenotypic characteristics and prognosis of inpatients with COVID-19 and diabetes: the CORONADO study. Diabetologia 2020;63:1500-15.

8 Cade BE, Dashti HS, Hassan SM, et al. Sleep apnea and COVID-19 mortality and hospitalization. Am J Respir Crit Care Med 2020;202:1462-4.

9 Maas MB, Kim M, Malkani RG. Obstructive sleep apnea and risk of COVID-19 infection, hospitalization and respiratory failure. Sleep Breath 2020:1-3.

10 Feuth T, Saaresranta T, Karlsson A, et al. Is sleep apnea a risk factor for Covid-19? findings from a retrospective cohort study. SMDIJ 2020;4:61-5.

11 Tufik S, Gozal D, Ishikura IA, et al. Does obstructive sleep apnea lead to increased risk of COVID-19 infection and severity? J Clin Sleep Med 2020;16:1425-6.

12 McSharry D, Malhotra A. Potential influences of obstructive sleep apnea and obesity on COVID-19 severity. J Clin Sleep Med 2020;16:1645.

13 Senaratna CV, Perret JL, Lodge CJ, et al. Prevalence of obstructive sleep apnea in the general population: a systematic review. Sleep Med Rev 2017;34:70-81.

14 Kohler M, Stradling JR. Mechanisms of vascular damage in obstructive sleep apnea. Nat Rev Cardiol 2010;7:677-85.

15 Young T, Skatrud J, Peppard PE. Risk factors for obstructive sleep apnea in adults. JAMA 2004;291:2013-6.

16 Kreivi H-R, Itäluoma T, Bachour A. Effect of ventilation therapy on mortality rate among obesity hypoventilation syndrome and obstructive sleep apnoea patients. ERJ Open Res 2020;6:00101-2019.

17 de Batlle J, Bertran S, Turino C, et al. Mortality in patients treated with continuous positive airway pressure at the population level. Am $J$ Respir Crit Care Med 2018;197:1486-8.

18 Strausz S, Ruotsalainen S, Ollila HM, et al. Genetic analysis of obstructive sleep apnoea discovers a strong association with cardiometabolic health. Eur Respir J 2020:2003091.

19 Apicella M, Campopiano MC, Mantuano M, et al. COVID-19 in people with diabetes: understanding the reasons for worse outcomes. Lancet Diabetes Endocrinol 2020;8:782-92.

20 Latest statistical releases. Available: https://www.tilastokeskus.fi/ index_en.html 